PAULO ALEXANDRE MARTIN

\title{
APLICAÇÃO DE TÉCNICAS DE CONTROLE PREDITIVO EM UMA COLUNA DE DESTILAÇÃO
}


PAULO ALEXANDRE MARTIN

\title{
APLICAÇÃO DE TÉCNICAS DE CONTROLE PREDITIVO EM UMA COLUNA DE DESTILAÇÃO
}

\author{
Tese apresentada à Escola \\ Politécnica da Universidade de São \\ Paulo para obtenção do título de \\ Doutor em Engenharia.
}

São Paulo 
PAULO ALEXANDRE MARTIN

\title{
APLICAÇÃO DE TÉCNICAS DE CONTROLE PREDITIVO EM UMA COLUNA DE DESTILAÇÃO
}

\author{
Tese apresentada à Escola \\ Politécnica da Universidade de São \\ Paulo para obtenção do título de \\ Doutor em Engenharia. \\ Área de Concentração: \\ Engenharia de Sistemas. \\ Orientador: \\ Prof. Dr. Fuad Kassab Junior.
}

São Paulo 
Aos meus pais e a todos que lutam para crescer profissionalmente com o objetivo de construir um país melhor. 


\section{AGRADECIMENTOS}

Meus especiais agradecimentos ao Prof. Dr. Fuad Kassad Junior, orientador desta tese, pelo apoio e pela ajuda durante a execução deste trabalho.

Especiais agradecimentos a Doutora Miriam Tvrzská de Gouvêa e ao Prof. Dr. Darci Odloak, cuja orientação e apoio foram essenciais para a execução deste trabalho.

Ao técnico Sidnei Moraes do bloco I da Escola de Engenharia Mauá, pela grande ajuda durante os ensaios com a coluna de destilação.

Agradecimentos ao Instituto Mauá de Tecnologia por ter permitido a utilização das instalações da planta piloto da Escola de Engenharia Mauá para o desenvolvimento de toda parte experimental, custeando as despesas com água e com a energia elétrica. 


\section{SUMÁRIO}

AGRADECIMENTOS

SUMÁRIO

LISTA DE FIGURAS

LISTA DE TABELAS

LISTA DE SÍMBOLOS

RESUMO

ABSTRACT

1 INTRODUÇÃO.

1

1.1 MOTIVAÇÕES E OBJETIVOS DO TRABALHO.................................. 1

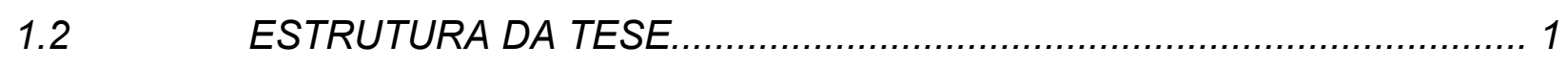

1.3 REVISÃO BIBLIOGRÁFICA

2 COLUNA DE DESTILAÇÃO.......................................................12

2.1 DESCRIÇÃO DO PROCESSO DE DESTILAÇÃO................................ 12

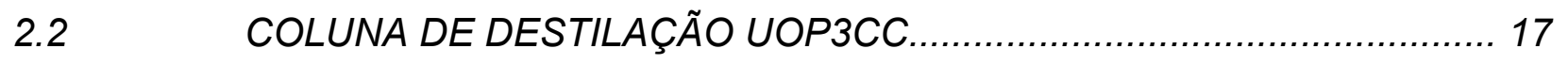

2.3 PAINEL DE CONTROLE DA COLUNA DE DESTILAÇÃO......................... 29

2.4 INSTRUMENTAÇÃO PARA A MEDIDA DA CONCENTRAÇÃO DE

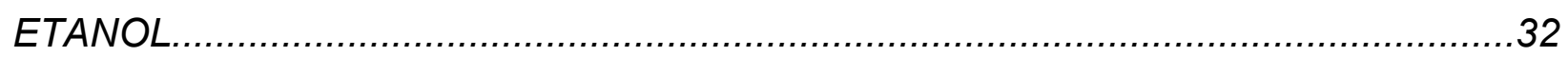

3 MODELAGEM DA COLUNA DE DESTILAÇÃO.......................... 38

3.1 INTRODUÇÃO AOS MODELOS LINEARIZADOS DA COLUNA DE

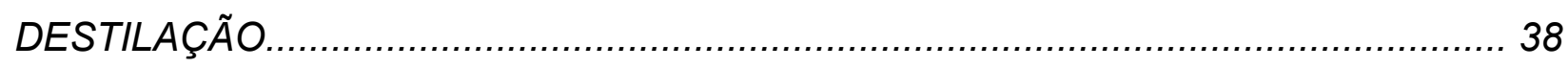

3.2 MODELOS LINEARIZADOS A TEMPO CONTÍNUO OBTIDOS. 42

3.2.1 MODELOS OBTIDOS A PARTIR DO ENSAIO R11.................... 42 


\section{SISTEMA DE CONTROLE PREDITIVO PARA COLUNA DE DESTILAÇÃO

4.1 CONTROLADOR PREDITIVO BASEADO EM MODELO. 50

4.2 CONTROLADOR PREDITIVO BASEADO EM MODELO ROBUSTO..... 52 4.3 CONTROLADOR PREDITIVO BASEADO EM MODELO COM COMPENSADOR. 53

\subsection{1}

INSERÇÃO DO CONTROLADOR PREDITIVO 69

4.4 IMPLEMENTAÇÃO DO RTO. 72

5 CONTROLADOR PREDITIVO ROBUSTO PARA SISTEMAS COM MÚLTIPLOS PÓLOS E INTEGRADOR.

5.1 CONTROLADOR PREDITIVO ROBUSTO MODIFICADO..................... 78 5.2 INSERINDO TEMPO MORTO NO SISTEMA A VARIÁVEIS DE ESTADO 99

5.3 DESENVOLVIMENTO DO CONTROLADOR DE MODELO NOMINAL. 102

5.4

CONTROLADOR PREDITIVO ROBUSTO ESTENDIDO. 112

5.4 .1 OBSERVADOR DE ESTADOS.

5.4 .2 INSERINDO ROBUSTEZ. 
6.3 MPC ROBUSTO

6.3.1 MPC ROBUSTO PARA PÓLOS MULTIPLOS..............................128

6.4 TESTES COM RTO

7 CONCLUSÕES. 135

REFERÊNCIAS BIBLIOGRÁFICAS 136 APÊNDICE 


\section{LISTA DE FIGURAS}

Figura 2.1 - Diagrama esquemático de uma simples coluna de destilação. 12

Figura 2.2 - Diagrama esquemático dos pratos da coluna de destilação. 14

Figura 2.3 - Curva de equilíbrio da mistura água-etanol. 15

Figura 2.4 - Diagrama para a estimação das frações molares no liquido e no vapor 16

Figura 2.5 - Foto perfil da coluna de destilação 17

Figura 2.6 - Diagrama esquemático da coluna de destilação UOP3CC 18

Figura 2.7 - Base da coluna de destilação com a indicação do visor para visualizar o nível de mistura. 19

Figura 2.8 - Trocador de calor. .20

Figura 2.9 - Tanques de alimentação. 20

Figura 2.10 - Bomba de alimentação 21

Figura 2.11 - Prato da coluna de destilação com o termopar para a medição de temperatura. 22

Figura 2.12 - Condensador. 23

Figura 2.13 - Decantador. .24

Figura 2.14 - Manômetro em U. .24

Figura 2.15 - Reservatório inferior. 25

Figura 2.16 - Reservatório superior e válvula de refluxo. 26

Figura 2.17 - Rotâmetro. 26

Figura 2.18 - Manômetro P1. 27

Figura 2.19 - Válvula V5. 27

Figura 2.20 - Reservatório para operação em destilação azeotrópica. 28

Figura 2.21 - Diagrama de blocos do sistema de aquisição de dados. 30

Figura 2.22 - Painel de controle da coluna de destilação. 30

Figura 2.23 - Desenho esquemático de um refratômetro. 33

Figura 2.24 - Refratômetro utilizado. 33

Figura 2.25 - Medida do índice de refração

Figura 2.26 - Curva do índice de refração em função da porcentagem mássica de etanol.

Figura 2.27 - Curva da densidade em função da porcentagem mássica de etanol..........35

Figura 2.28 - Picnômetro utilizado. 
Figura 2.29 - Diagramas de fase da mistura água-etanol

Figura 3.1 - Resposta ao degrau da temperatura T1 e seu respectivo modelo para uma variação de $+90 \mathrm{~W}$ de potência em torno do ponto de operação $R=70 \%, P=500$ W.

Figura 3.2 - Resposta ao degrau da temperatura T4 e seu respectivo modelo para uma variação de $+90 \mathrm{~W}$ de potência em torno do ponto de operação $R=70 \%, P=500$ W.

Figura 3.3 - Resposta ao degrau da temperatura T1 e seu respectivo modelo para uma variação de $+90 \mathrm{~W}$ de potência em torno do ponto de operação $R=70 \%, P=700$ W.

Figura 3.4 - Resposta ao degrau da temperatura T4 e seu respectivo modelo para uma variação de $+90 \mathrm{~W}$ de potência em torno do ponto de operação $R=70 \%, P=700$ W.

Figura 3.5 - Resposta ao degrau da temperatura T1 e seu respectivo modelo de pólos distintos para uma variação de $+90 \mathrm{~W}$ de potência em torno do ponto de operação $\mathrm{R}=$ $70 \%, P=500 \mathrm{~W}$.

Figura 3.6 - Resposta ao degrau da temperatura T4 e seu respectivo modelo de pólos distintos para uma variação de $+90 \mathrm{~W}$ de potência em torno do ponto de operação $\mathrm{R}=$ $70 \%, P=500 \mathrm{~W}$

Figura 4.1 - Integração entre o controlador MPC e o controlador. .54

Figura 4.2 - Integração entre o controlador MPC e o controlador com um pós compensador que diminui os laços de controle .55

Figura 4.3 - Sistema de controle com "Preditor de Smith". .58

Figura 4.4 - Diagrama de blocos final da primeira malha de realimentação. 60 Figura 4.5 - Diagrama de bode de $\mathrm{H}(\mathrm{s})$ para todos os pontos de operação da planta.

Figura 4.6 - diagrama de blocos de mostra o mecanismo de adaptação do avançador atrasador. 64

Figura 4.7 - diagrama de blocos de mostra o mecanismo de adaptação dos preditores de segunda ordem

Figura 4.8 - diagrama de blocos que mostra o mecanismo de adaptação do controlador $\mathrm{PI}$

Figura 4.9 - Resposta ao degrau de $\mathrm{H}(\mathrm{s})$ compensado por $\mathrm{Gc}_{11}(\mathrm{~s})$ para todos os pontos de operação da planta. 
Figura 4.10 - Resposta ao degrau de $\mathrm{G}_{22}(\mathrm{~s})$ compensado por $\mathrm{Gc}_{22}(\mathrm{~s})$ para todos os pontos de operação da planta.

Figura 4.11 - Resposta do sistema com MPC convencional para T1'(s) com wy=0,6 e $\mathrm{wu}=1,0$.

Figura 4.12 - Resposta do sistema com MPC robusto para T1'(s) com wy=0,6 e $\mathrm{wu}=1,0$. 70

Figura 4.13 - Resposta do sistema com MPC convencional para T4(s) wy=0,4 e $\mathrm{wu}=1,0$.

Figura 4.14 - Resposta do sistema com MPC robusto para T4(s) com wy=0,5 e $\mathrm{wu}=1,0$. .71

Figura 4.15 - Implementação de um RTO no sistema. .72

Figura 4.16 - Sistema de controle com controlador preditivo robusto. .74

Figura 4.17 - Sistema de controle com o controlador preditivo robusto no ponto de operação $P=500 \mathrm{~W}$ e $\mathrm{R}=50 \%$ com $\mathrm{q}_{11}=0,7, \mathrm{q}_{22}=0,7, \mathrm{r}_{11}=0,5$ e $\mathrm{r}_{22}=0,5$. Os valores de setpoints são $1^{\circ} \mathrm{C}$ e $0,7^{\circ} \mathrm{C}$ para $\mathrm{T} 1$ e $\mathrm{T} 4$ respectivamente..... 75

Figura 4.18 - Sistema de controle com o controlador preditivo robusto no ponto de operação $P=500 \mathrm{~W}$ e $\mathrm{R}=70 \%$ com $\mathrm{q}_{11}=0,7, \mathrm{q}_{22}=0,7, \mathrm{r}_{11}=0,5$ e $\mathrm{r}_{22}=0,5$. Os valores de setpoints são $2^{\circ} \mathrm{C}$ e $1^{\circ} \mathrm{C}$ para $\mathrm{T} 1$ e $\mathrm{T} 4$ respectivamente. 75

Figura 4.19 - Sistema de controle com o controlador preditivo robusto no ponto de operação $P=500 \mathrm{~W}$ e $\mathrm{R}=30 \%$ com $\mathrm{q}_{11}=0,5, \mathrm{q}_{22}=0,5, \mathrm{r}_{11}=0,5$ e $\mathrm{r}_{22}=0,2$. Os valores de setpoints são $2^{\circ} \mathrm{C}$ e $1,1^{\circ} \mathrm{C}$ para $\mathrm{T} 1$ e $\mathrm{T} 4$ respectivamente..... 76

Figura 4.20 - Sistema de controle com o controlador preditivo robusto no ponto de operação $P=500 \mathrm{~W}$ e $\mathrm{R}=70 \%$ com $\mathrm{q}_{11}=0,5, \mathrm{q}_{22}=0,5, \mathrm{r}_{11}=0,5$ e $\mathrm{r}_{22}=0,2$. Os valores de setpoints são $2^{\circ} \mathrm{C}$ e $1,2^{\circ} \mathrm{C}$ para $\mathrm{T} 1$ e $\mathrm{T} 4$ respectivamente

Figura 5.1 - Simulação para o ponto de operação $P=900 \mathrm{~W}$ e R $=50 \%$ com com $q_{11}=0,5, q_{22}=0,5, r_{11}=0,5$ e $r_{22}=0,2$. Os valores de setpoints são $2^{\circ} \mathrm{C}$ e $1^{\circ} \mathrm{C}$ para $\mathrm{T} 1$ e $\mathrm{T} 4$ respectivamente. 120

Figura 5.2 - Simulação para o ponto de operação $P=700 \mathrm{~W}$ e R $=50 \%$ com com $q_{11}=0,5, q_{22}=0,5, r_{11}=0,5$ e $r_{22}=0,2$. Os valores de setpoints são $2^{\circ} \mathrm{C}$ e $0^{\circ} \mathrm{C}$ para $\mathrm{T} 1$ e $\mathrm{T} 4$ respectivamente

Figura 5.3 - Simulação para o ponto de operação $P=500 \mathrm{~W}$ e $R=50 \%$ com com $\mathrm{q}_{11}=0,5, \mathrm{q}_{22}=0,5, \mathrm{r}_{11}=0,5$ e $\mathrm{r}_{22}=0,2$. Os valores de setpoints são $2^{\circ} \mathrm{C}$ e $1,1^{\circ} \mathrm{C}$ para $\mathrm{T} 1$ e $\mathrm{T} 4$ respectivamente. 
Figura 5.4 - Simulação para o ponto de operação $P=700 \mathrm{~W}$ e $\mathrm{R}=30 \% \mathrm{com}$ com $\mathrm{q}_{11}=0,7, \mathrm{q}_{22}=0,7, \mathrm{r}_{11}=0,5$ e $\mathrm{r}_{22}=0,5$. Os valores de setpoints são $1^{\circ} \mathrm{C}$ e $0^{\circ} \mathrm{C}$ para $\mathrm{T} 1$ e $\mathrm{T} 4$ respectivamente.

Figura 6.1 - Resultado experimental do MPC com compensador para T1 123

Figura 6.2 - Resultado experimental do MPC com compensador para T4. 124

Figura 6.3 - Resultado experimental do MPC robusto com compensador para T1 ......125

Figura 6.4 - Resultado experimental do MPC robusto com compensador para T4......125

Figura 6.5 - Resultado experimental do MPC robusto com pólos distintos para $\mathrm{T} 1$ 126

Figura 6.6 - Resultado experimental do MPC robusto com pólos distintos para T4.

Figura 6.7 - Segundo resultado experimental do MPC robusto com pólos distintos para $\mathrm{T} 1$ 127

Figura 6.8 - Segundo resultado experimental do MPC robusto com pólos distintos para T4. 128

Figura 6.9 - Resultado experimental do MPC robusto com pólos múltiplos para $\mathrm{T} 1$. 128

Figura 6.10 - Resultado experimental do MPC robusto com pólos múltiplos para T4. 129

Figura 6.11 - Diagrama de blocos do sistema com RTO. 131

Figura 6.12 - Fluxograma do algoritmo de busca exaustiva. 132

Figura 6.13 - Teste com RTO no controlador robusto para pólos em multiplicidade em T1 para Cel $=0,38 \mathrm{R} \$ / \mathrm{kWh}$. 134

Figura 6.14 - Teste com RTO no controlador robusto para pólos em multiplicidade em $\mathrm{T} 4$ para Cel $=0,38 \mathrm{R} \$ / \mathrm{kWh}$. 134 


\section{LISTA DE TABELAS}

Tabela 2.1 - Características técnicas dos equipamentos utilizados na coluna de

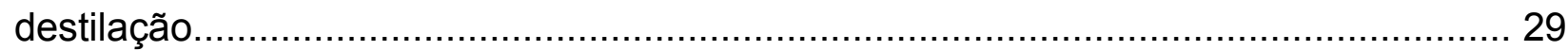

Tabela 2.2 - Relação de sinais de entrada e saída na interface de $\mathrm{I} / \mathrm{O}$ 1 ..........................31

Tabela 2.3 - Relação de sinais de entrada e saída na interface de $\mathrm{I} / \mathrm{O} 2 \ldots \ldots \ldots \ldots \ldots \ldots \ldots . . . . . .32$

Tabela 3.1 - Pontos de operação para o levantamento dos modelos da coluna de

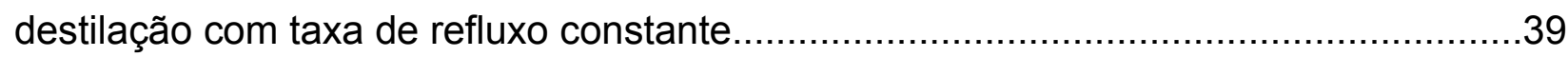

Tabela 3.2 - Pontos de operação para o levantamento dos modelos da coluna de destilação com potência do aquecedor constante......................................................39 Tabela 3.3 - Modelos lineares obtidos para os diferentes pontos de

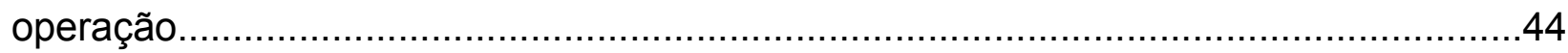

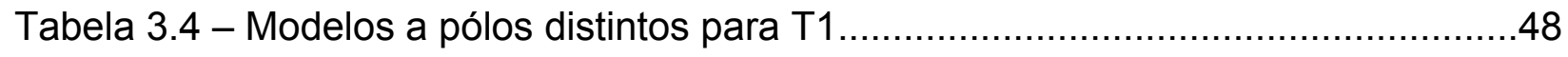

Tabela 3.5 - Modelos a pólos distintos para T4 .....................................................

Tabela 4.1 - Valores de sintonia do avançador-atrasador para todos os pontos de

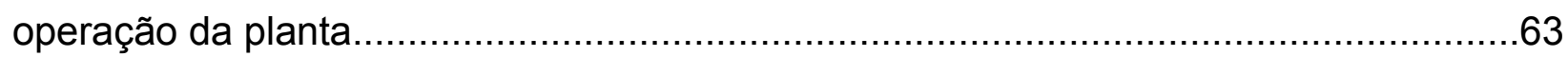

Tabela 4.2 - Valores de sintonia do controlador PI para todos os pontos de operação da planta 66

Tabela 6.1 - Testes com RTO 133 


\section{LISTA DE SÍMBOLOS}

$\mathrm{G}_{\mathrm{ij}}(\mathrm{s})$ : função de transferência da planta que relaciona a saída i com a entrada j.

$\mathrm{K}_{\mathrm{ij}}$ : ganho da função de transferência Gij(s) da planta.

$\tau_{\mathrm{ij}}$ : constante de tempo da função de transferência Gij(s) da planta.

$T_{i j:}$ : tempo morto da função de transferência Gij(s) da planta.

$\mathrm{g}(\mathrm{t})$ : modelo utilizado.

$f(t)$ : resposta do processo.

$\eta(t)$ : peso aplicado para cada instante de tempo.

$\varepsilon$ : erro de estimação na modelagem.

$\mathrm{T}_{1}(\mathrm{~s})$ : temperatura $\mathrm{T}_{1}$ no domínio $\mathrm{s}$.

$\mathrm{T}_{4}(\mathrm{~s})$ : temperatura $\mathrm{T}_{4}$ no domínio $\mathrm{s}$.

$P(s)$ : potência do aquecedor no domínio s.

$R(s)$ : taxa de refluxo no domínio $s$.

$\mathrm{J}$ : função custo do MPC convencional.

$\mathrm{w}(\mathrm{t})$ : trajetória de referência do MPC convencional.

$y(t)$ : saída da planta do MPC convencional.

$\mathrm{u}(\mathrm{t})$ : sinal de controle do MPC convencional.

$\delta(j)$ : função penalizadora do erro de trajetória do MPC convencional.

$\lambda(\mathrm{j})$ : função penalizadora do esforço de controle do MPC convencional.

$u_{\text {min }}$ : valor mínimo admissível de u.

umax: valor máximo admissível de $u$.

$y_{\text {min }}$ : valor mínimo admissível de $y$.

$y_{\max }$ : valor máximo admissível de $y$.

$\Delta \mathrm{u}_{\text {min }}$ : valor mínimo admissível de $\Delta \mathrm{u}$.

$\Delta \mathrm{u}_{\text {max }}$ : valor máximo admissível de $\Delta \mathrm{u}$.

R: matriz penalizadora do erro de trajetória do MPC multivariável.

Q: matriz penalizadora do esforço de controle do MPC multivariável.

$V_{k}$ : função custo do MPC robusto.

$\theta$ : vetor que contém todos as matrizes $\mathrm{A}$ e $\mathrm{B}$ que descrevem os modelos da planta.

$\mathrm{G}(\mathrm{s})$ : matriz de transferência da planta.

$\mathrm{D}(\mathrm{s})$ : pós-compensador diagonalizador da planta.

$\Delta_{\mathrm{ij}}$ : tempo morto da função de transferência $\mathrm{G}_{\mathrm{ij}}(\mathrm{s})$.

$\mathrm{U}_{1}(\mathrm{~s})$ : potência do aquecedor no domínio s. 
$\mathrm{U}_{2}(\mathrm{~s})$ : taxa de refluxo no domínio $\mathrm{s}$.

$\varphi_{\mathrm{m}}$ : máximo ganho de fase do avançador.

$\omega_{m}$ : freqüência onde ocorre o máximo ganho de fase do avançador.

$\mathrm{Gc}_{11}(\mathrm{~s})$ : compensador para $\mathrm{T}_{1}(\mathrm{~s})$.

$\mathrm{Gc}_{22}(\mathrm{~s})$ : compensador para $\mathrm{T}_{4}(\mathrm{~s})$.

$\mathrm{C}_{\mathrm{el}}$ : custo da energia elétrica em $\mathrm{R} \$ / \mathrm{J}$.

$\mathrm{y}_{10}$ : concentração de etanol no destilado no topo da coluna.

$\mathrm{L}_{\mathrm{d}}$ : lucro do destilado em $\mathrm{R} \$$ /litro.

$\mathrm{k}_{\mathrm{d}}$ : ganho que relaciona a vazão de destilado com a potência do aquecedor.

$\mathrm{k}_{\mathrm{ij}}$ : número de pólos em multiplicidade da função de transferência que relaciona a saída i com a entrada $\mathrm{j}$.

$p_{i j}$ : número de pólos distintos da função de transferência que relaciona a saída i com a entrada j.

$\mathrm{S}_{\mathrm{ij}}(\mathrm{t})$ : resposta ao degrau unitário da função de transferência $\mathrm{G}_{\mathrm{ij}}(\mathrm{s})$.

$\left[\mathrm{x}_{\mathrm{i}}^{\mathrm{i}}\right]$ : estado do ganho estático de $\mathrm{G}_{\mathrm{ij}}(\mathrm{s})$.

$\left[\mathrm{x}_{\mathrm{i}}^{\mathrm{i}}\right]$ : estado do integrador de $\mathrm{G}_{\mathrm{ij}}(\mathrm{s})$.

$\left[\mathrm{x}_{\mathrm{i}}^{\mathrm{i}}\right]$ : vetor de estados dos pólos em multiplicidade de $\mathrm{G}_{\mathrm{ij}}(\mathrm{s})$.

$\left[\mathrm{x}_{\mathrm{i}}^{\mathrm{f}}\right]$ : vetor de estados dos pólos distintos de $\mathrm{G}_{\mathrm{ij}}(\mathrm{s})$.

$A_{i j}$ : matriz de inércia do sistema a variáveis de estado de $G_{i j}(s)$.

$B_{i j}$ : matriz de ganhos do sistema a variáveis de estado de $G_{i j}(s)$.

$C_{i j}$ : matriz de saída do sistema a variáveis de estado de $G_{i j}(s)$.

$C_{i j}(t)$ : funções no tempo que são a predição da saída para o sistema $G_{i j}(s)$.

$A_{i j}$ : matriz de inércia dos pólos em multiplicidade do sistema a variáveis de estado de $\mathrm{G}_{\mathrm{ij}}(\mathrm{s})$.

$A_{i j}^{f}$ : matriz de inércia dos pólos distintos do sistema a variáveis de estado de $\mathrm{G}_{\mathrm{ij}}(\mathrm{s})$.

$\mathrm{B}_{\mathrm{ij}}^{\mathrm{d}}$ : matriz de ganhos dos pólos em multiplicidade do sistema a variáveis de estado de $\mathrm{G}_{\mathrm{ij}}(\mathrm{s})$.

$B_{i j}^{f}$ : matriz de ganhos dos pólos distintos do sistema a variáveis de estado de $G_{i j}(s)$.

$\left.\mathrm{C}_{i j}{ }^{\mathrm{d}} \mathrm{t}\right)$ : funções no tempo que são a predição da saída relativa aos pólos em multiplicidade para o sistema $\mathrm{G}_{\mathrm{ij}}(\mathrm{s})$.

$\mathrm{C}_{\mathrm{ij}}^{f}(\mathrm{t})$ : funções no tempo que são a predição da saída relativa aos pólos distintos para o sistema $\mathrm{G}_{\mathrm{ij}}(\mathrm{s})$.

ny: número de saídas do sistema MIMO.

nu: número de entradas do sistema MIMO.

$\mathrm{x}$ : vetor de estados do sistema MIMO. 
$\overline{\mathrm{A}}$ : matriz de inércia do sistema MIMO.

$\bar{B}$ : matriz de ganhos do sistema MIMO.

$\overline{\mathrm{C}}$ : matriz de saída do sistema MIMO.

$\Psi(\mathrm{t})$ : matriz que contém as funções no tempo que dão a predição das saídas do sistema MIMO.

$\phi_{\mathrm{ij}}(\mathrm{t})$ : submatriz que contém as funções no tempo que são a predição da saída do sistema representado por $\mathrm{G}_{\mathrm{ij}}(\mathrm{s})$.

$\hat{\mathrm{X}}^{\mathrm{s}}$ : vetor de estados dos ganhos estáticos modificado.

$\hat{x}^{\prime}$ : vetor de estados dos integradores modificado.

$\hat{\mathrm{x}}^{\mathrm{d}}$ : vetor de estados dos pólos em multiplicidade modificado.

$\hat{\mathrm{X}}^{\mathrm{f}}$ : vetor de estados dos pólos distintos modificado.

$\hat{\mathrm{X}}$ : vetor de estados do sistema MIMO modificado.

$\hat{A}^{d}$ : matriz de inércia relativa aos pólos em multiplicidade modificada.

$\hat{A}^{f}$ : matriz de inércia relativa aos pólos distintos modificada.

$\hat{A}$ : matriz de inércia modificada.

$\hat{B}^{s}$ : matriz de ganho modificada relativa aos estados de ganho estático do sistema.

$\hat{B}^{\prime}$ : matriz de ganho modificada relativa aos estados dos integradores do sistema.

$\hat{\mathrm{B}}^{\mathrm{d}}$ : matriz de ganho modificada relativa aos pólos em multiplicidade do sistema.

$\hat{B}^{f}$ : matriz de ganho modificada relativa aos pólos distintos do sistema.

$\hat{\mathrm{C}}$ : matriz de saída modificada do sistema.

$\hat{\mathrm{C}}(\mathrm{t})$ : matriz modificada das funções no tempo que dão a predição das saídas do sistema.

np: horizonte de predição.

$\mathrm{m}$ : horizonte das ações de controle.

$\tilde{\mathrm{X}}$ : vetor de estados englobando o efeito do tempo morto do sistema.

Á: matriz de inércia do sistema englobando o efeito do tempo morto.

$\tilde{B}$ : matriz de ganhos do sistema englobando o efeito do tempo morto.

Cे: matriz de saída do sistema englobando o efeito do tempo morto.

ne: número de estados do sistema englobando o efeito do tempo morto.

$\tilde{\Psi}^{\prime}(\mathrm{t})$ : funções no tempo que dão a predição das saídas relativas ao efeito dos pólos integradores considerando o efeito do tempo morto.

$\tilde{\Psi}^{\mathrm{d}}(\mathrm{t})$ : funções no tempo que dão a predição das saídas relativas ao efeito dos pólos em multiplicidade considerando o efeito do tempo morto. 
$\tilde{\Psi}^{f}(t)$ : funções no tempo que dão a predição das saídas relativas ao efeito dos pólos distintos considerando o efeito do tempo morto.

$\mathrm{S}_{\mathrm{h}}$ : submatrizes da matriz de ganho $\tilde{\mathrm{B}}$.

$\mathrm{y}_{1}^{\text {sp }}$ : vetor de setpoints do sistema.

$\ddot{A}$ : matriz de inércia do sistema sem os efeitos dos estados de ganho estático e estados dos integradores englobando o efeito do tempo morto.

$\mathrm{H}_{1}$ : matriz Hessiana da função objetivo do controlador nominal.

$\mathrm{H}_{\mathrm{e}}$ : matriz Hessiana da função objetivo do controlador estendido.

$\delta^{s}$ : variável de folga para os estados relativos ao ganho estático do sistema.

$\delta^{\prime}$ : variável de folga para os estados relativos aos integradores do sistema.

$\mathrm{K}_{\mathrm{F}}$ : ganho do observador de estados.

$\mathrm{T}_{\text {off: }}$ temperatura em $\mathrm{T}_{1}$ relativa ao ponto de operação $\mathrm{P}=500 \mathrm{~W}$ e $\mathrm{R}=70 \%$.

$\mathrm{g}_{11}$ : ganho estático da potência do aquecedor em $\mathrm{T}_{1}$.

$\mathrm{g}_{12}$ : ganho estático da taxa de refluxo em $\mathrm{T}_{1}$. 


\section{RESUMO}

Este trabalho apresenta todos os passos para a implementação de técnicas de controle preditivo em uma coluna de destilação. Inicialmente a tese introduz basicamente o funcionamento e a meta do processo de destilação. Modelos linearizados em tempo continuo da coluna de destilação são obtidos a partir de ensaios experimentais da coluna em diferentes pontos de operação. Com base nestes modelos, várias topologias de controladores preditivos baseados em modelo são implementadas. Um otimizador em tempo real é integrado aos controladores preditivos para a redução do custo operacional da planta. Resultados simulados e resultados experimentais de todas as topologias de controladores preditivos estudados são apresentados. 


\begin{abstract}
This work presents all the steps to the implementation of predictive control techniques in a distillation column. First the thesis basically introduces the working and the goal of the distillation process. Linearized models in continuous time of the distillation column are obtained from experimental tests of the column in different operating points. Based on this models, several model based predictive controllers topologies are implemented. A real time optimizer is integrated with the predictive controllers to the reduction of the plant operational cost. Simulated results and experimental results of all studied predictive controllers topologies are presented.
\end{abstract}

